\title{
Study on Operational Risk Management of Neural Network Commercial Bank
}

\author{
Lijuan Liü, a \\ ${ }^{1}$ Shaanxi Institute of International Trade \& Commerce, Xianyang, Shaanxi, 712046 \\ ${ }^{\mathrm{a}}$ email
}

Keywords: Commercial Banks, Operational Risk, Neural Network

\begin{abstract}
Operational risk is a major component of commercial bank risk management. With the continuous development of society, but also gradually facing the globalization of financial close, while commercial banks have also faced with increasingly complex operational risk. This requires commercial banks to effectively manage operational risk, if not timely response, not only has a huge impact on the operations and competitiveness of commercial banks, commercial banks and even lead to death.
\end{abstract}

\section{Introduction}

In the new era of economic system, the commercial banks of the financial center, which have played an irreplaceable role in financial intermediation and regulation of social and other aspects of supply demand balance. At the same time it is also accompanied by a number of risks, such as credit risk, operational risk. In 1997, the Basel Committee on Banking Supervision officially announced the commercial bank risk is divided into eight categories, including operational risks due to its extensive and immeasurable become the main objective of the current study.

\section{The Purpose and Significance}

Commercial Bank Operational Risk Measurement and Management Research management to effectively improve the management level and economic efficiency of commercial banks have a great impact. In recent years, many European countries have spared no effort in research and measurement of operational risk, in order to effectively conserve capital. Through continuous accumulation of research, the mechanism of operational risk in some countries has been maturing. In comparison with foreign countries, currently operational risk management level is relatively low, which we need to increase research in order to establish a sound system.

First, we need the concept of traditional commercial banks operational risk start, it will be combined with the use of some aspects of the system theory, process theory. To determine the risk measure in targeting operational risk management, and lay a solid foundation for future development. Secondly, we want to build the model by effectively combining fuzzy control and neural networks. A measure of the operational risk loss of reasonable evaluation. Through research to improve. Finally, at the operational risk measurement combined with advanced financial technology, the bank operational risk effectively integrated management.

Operational risk management of commercial banks for the development and operation of commercial banks have a huge impact, so risk measurement depth practical significance is the inevitable trend. Which is mainly reflected in two aspects (1) to improve the management of operational risk. Compared to the West is relatively mature approach to risk management, operational risk management of the short time since the study, theoretical shortages, remained stagnant. Some foreign banks not copy management system is also facing environmental factors, cultural factors and other issues. According to China's actual situation today is to study their own operational risk management theory is imperative trend. Only then is the theory of operational risk management is more systematic. A huge change for the Risk Management of China Commercial Bank. (2) strengthen the operational risk management, improve the internal management level. In modern commercial bank management risk management has a central position. Because today's 
corporate financial fragility and lack of supervision, etc., resulting in commercial banks there is a huge risk. We can strengthen the commercial bank process management inside, from inside to outside monitoring. Provide a strong basis for the commercial bank risk management.

\section{The Commercial Banks Operational Risk Measurement Objectives and Constraints}

Operational risk is a relatively complicated process, which requires us to regularly evaluate the operation to reduce the risk of loss. Thereby reducing the impact on business caused by operational risk. Achieve the purpose, as shown below [1].

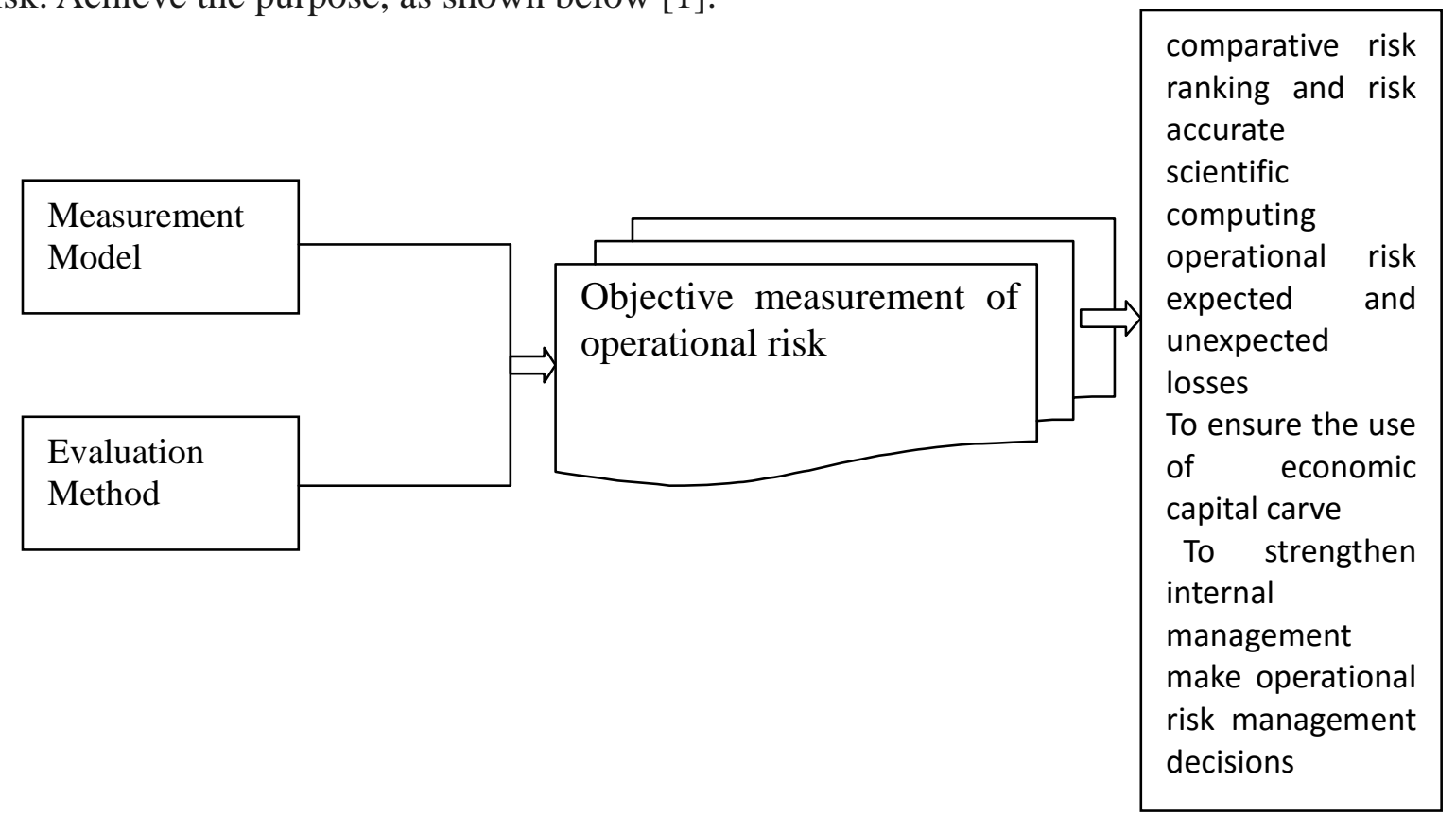

Risk Ranking. By operational risk measure can effectively between different departments operate relatively risk, and do a very good sort. This allows for real-time status of operational risk understanding; by measurement of operational risk can effectively allow commercial banks to carry out a reasonable allocation of risk management, and to take appropriate management techniques.

A reasonable allocation of economic capital. By operational risk measure, the state can effectively assess the risk of loss, so that you can measure will be developed into requirements amount. In order to protect the capital adequacy. Bring great convenience for operators of commercial banks.

Calculate the risk of loss. Through precise quantification of operational risk, operational risk can effectively loss calculation. This cost can be calculated in advance, thereby reducing the risk of loss.

The establishment of risk management policy is primarily a measure of operational risk based. Precise measure allows management to develop a reasonable strategy, in order to make scientific investment to bring security to the normal operation of commercial banks.

Accurate measure directly affects the operational risk management, operational risk management and effective at the same time can improve the operating results of commercial banks. Only operating results improved to motivate each employee, so that you can make them more control over operational risk, reduce the risk of loss. Encourage all employees are in progress, in order to improve risk management level make a significant contribution.

Due to the characteristics of the operating system itself, the limiting factor is greater in the operational risk measurement, which increased the metric method. The main limiting factors are the following: (1) the coverage is too large and thus lead to a lot of favoritism and other behavior. But also the inevitable natural disasters. (2) qualitative factors affected by the policy, legal, environmental and other effects too. (3) definition and classification of different metrics for indirect losses affecting its objectivity. (4) measurement of operational risk experience and limited data, which resulted in low-rate losses. At the same time unable to carry out effective inspection results, these factors have led to China must increase research efforts in the above measurement of 
operational risk.

\section{Construction of BP Neural Network Predictive Model}

BP neural network algorithm. BP neural network is a multi-layer anti-pass calculation error feed-forward neural network. Consisting of an input layer, one or more hidden layers and an output layer. Network structure mainly consists of three parts, (1) Connection (2) network layer (3) the number of layers of nodes. As has been proved theoretically, a three-layer BP neural network on any mapping have a good effect. So we use the BP neural network model to a reasonable measure of commercial banks operational risk.

According to the root of the operating risk of commercial banks and encourage the formation, we can through financial and financial indicators to measure effectively. We recommend combining the "New Basel Capital Accord," the select nine risk indicators. This makes it representative, sensitivity and timeliness characteristics. So that we can better observe the effect. We use X (x1, x2, .., x9) to represent the selected indicator is our input nodes, hidden layer we use Y (y1, y2, y3, y4, y5) to represent. At the same time with $\mathrm{O}(\mathrm{o} 1)$ to indicate the value of commercial banks operational risk, while its output layer node is the only entire model.

Select the hidden layer node is more complex, but also affect the performance of its network is relatively large. For the three-layer neural network, we can use this formula to determine the best hidden nodes. Wherein $\mathrm{m}, \mathrm{n}$, representing the input layer and output layer unit, $\mathrm{c}$ is a constant. After repeated tests that give input nodes and output nodes are each 1 and 9, the hidden layer nodes is 5, the neural network convergence function, so in this case the lowest rate of false positives.

If the input layer node to node weights hidden layer with V1 - V9, hidden layer to the output layer node weights represented by W1-W5. For the hidden layer has the following formula :), $\mathrm{j}=$ $1,2,3,4,5$ for the output layer there: where $\mathrm{f}(\mathrm{x})$ for the excitation function: It can draw the network structure of the measure [2].

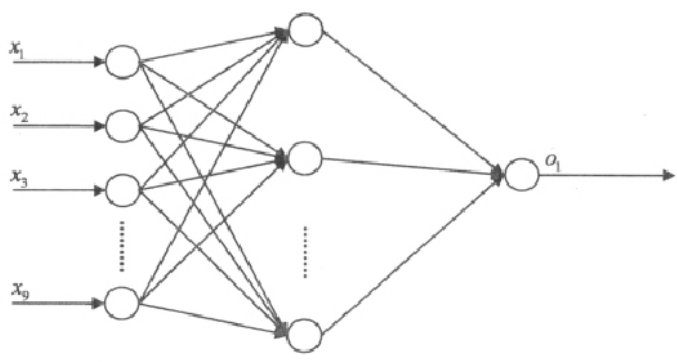

The so-called neural network learning, in fact, the individual weights of the adjustment process, the only constant on the right to an effective value of adjustments in order to constantly reduce the error, and therefore adjust the amount of the negative gradient and the error is proportional relationship must, if errors. According to BP algorithm, we were able to model the right to adjust the value of the formula [3].

In the two formulas $\mathrm{j}=1,2,3,4,5$. Where, $\mathrm{d} 1$ is the desired output value for the learning rate. Is the use of hair all over the automatic adjustment based on the output of the error, mainly in order to speed up the convergence. Where $\alpha$ is the adjustment step, $(0,1)$ value, Ep (n) is the desired output error and the calculated output. When the error precision $\mathrm{O} 1$ and $\mathrm{d} 1 \mathrm{can}$ meet the requirements, we stop to adjust the weights, the process ends. Obtained by calculating the neural network has a huge impact on the commercial banks operational risk management. We can calculate the economic costs to the budget in advance, so that we can effectively avoid operational risk. Thus the normal operation of commercial banks to contribute.

\section{Conclusion}

My general lack of modern commercial bank operating risk loss of data, so how to choose the right mode for the current measure of the operational risk management of commercial banks is particularly important. Operational risk proposed measure neural network of commercial banks 
operational risk measurement system has a great influence. Able to avoid significant loss of data required for operational risk measurement also has some practical value, but also a strong operability.

\section{References}

[1] Feng Jichuang, Li Jianping, Gao Lijun. Commercial bank operating risk measurement model selection analysis [J]. International Financial Research, 2011 (8), 88-96。

[2] Sima Zerong, Cai Chen, Li Jianping. based on the distribution of operational risk measurement [J]. systems engineering theory and practice, 2011,31 (12): 2321-2327.

[3] Shi Wujiang, Feng Jichuang. nonparametric estimation method operational risk measure: based on the maximum entropy principle [J]. Economic Industrial Technology, 2011, 30 (7), 115-122. 